\title{
Soft X-ray Emission Spectroscopy on Chemical States of 3D-Transition Metal Elements with SEM
}

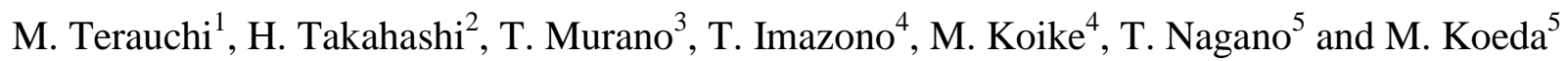 \\ 1. IMRAM, Tohoku University, 2-1-1 Katahira, Aoba-ku, Sendai 980-8577, Japan \\ 2. Global business promotion division, JEOL Ltd., 2-1-1 Otemachi, Chiyoda-ku, Tokyo 100-0004, Japan \\ 3. SA Technical Development Division, JEOL Ltd., 3-1-2 Musashino, Akishima, Tokyo 196-8558, Japan \\ 4. Quantum Beam Science Center, Japan Atomic Energy Agency, 8-1-7 Umemidai, Kizu 619-0215, Japa. \\ 5. Device Department, Shimadzu Corp., 1 Nishinokyo-Kuwabaracho, Nakagyo-ku, Kyoto 604-8511, Japan
}

Soft X-ray emission spectrometer for 50-4000 eV had been attached to a SEM [1], and has been applied to 3dtransiton metals and compounds including battery cathode materials. X-rays originate from electronic transitions from valence bands (VB, bonding electron states) to inner-shell electron levels inform us of energy states of bonding electrons. Thus, L-emissions due to transitions of 3d valence electrons to 2p core-hole are very important to assign the chemical states of 3d-transiton metal elements. Furthermore, charge state is also important to discuss the physical property because of the amount of 3d electrons closely related to magnetism and conductivity of transition metal compounds.

L $\alpha, \beta$ emissions attributed to $3 \mathrm{~d}_{5 / 2,3 / 2} \rightarrow 2 \mathrm{p}_{3 / 2}$ and $3 \mathrm{~d}_{3 / 2} \rightarrow 2 \mathrm{p}_{1 / 2}$ transitions, respectively, are suitable to probe valence states (bonding states). On the other hand, Ll, $\eta$ emissions are due to $3 \mathrm{~s}_{1 / 2} \rightarrow 2 \mathrm{p}_{3 / 2}$ and $3 \mathrm{~s}_{1 / 2}$ $\rightarrow 2 p_{1 / 2}$ transitions, respectively. The 3 s and $2 p$ electron levels of 3d-elements are core states. Thus, Ll, $\eta$ lines are appropriate to observe a change of binding energy of core electrons (chemical shift).

$\mathrm{TiO}_{2}$ is an important photocatalytic material. Figure 1 shows L $\alpha, \beta$ emission spectra obtained from bulk $\mathrm{TiO}_{2}$ specimens having various morphology, i.e., rutile, anatase, and brookite, by use of the SXES-SEM instrument with a grating for 300-2200 eV developed by us [2]. A spectrum of metal-Ti is also shown for comparison. In a simple ionic model, $\mathrm{TiO}_{2}$ is considered as $\mathrm{Ti}^{4+} \mathrm{O}^{2-}{ }_{2} \cdot \mathrm{Ti}^{4+}$ ion has no $3 \mathrm{~d}$ electron. However, $\mathrm{L} \alpha, \beta$ emissions are observed for each $\mathrm{TiO}_{2}$ material, reflecting covalent bonding between $\mathrm{Ti}$ and $\mathrm{O}$ atoms. Characteristic profile structures indicated by vertical lines vary for different $\mathrm{TiO}_{2}$ materials due to particular band structures, which are attributed to different crystal structures. When comparing the peak positions of $\mathrm{L} \alpha, \beta$ spectra, those of $\mathrm{TiO}_{2}$ are located at the lower energy side than those of metal-Ti. They are opposite shifts for $\mathrm{Ti}^{4+}$ ion. This means that $\mathrm{L} \alpha, \beta$ intensity profile influenced by the density of states of VB is not appropriate to detect chemical shift.

Figure 2 shows $\mathrm{Ll}, \eta$ emission spectra of bulk $\mathrm{TiO}_{2}$, rutile, anatase, and brookite along with metal-Ti. All data points of the respective spectra were obtained at the same time with the corresponding spectra shown in Figure 1, because the spectrometer with the present grating allows us a parallel detection for 300-2200 eV. As Ll, $\eta$ emissions are caused by transitions between inner-shell levels, intensity profiles

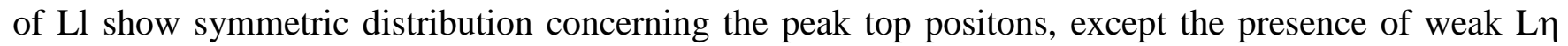
intensity on the high energy side. It is clearly seen that $\mathrm{Ll}$ peaks of $\mathrm{TiO}_{2}$ materials are positioned at higher energy side than that of metal-Ti. These shifts of $\mathrm{TiO}_{2}$ materials are reasonable for cation chemical shift. Furthermore, shift amounts of rutiel and anatase are almost the same, but that of brookite is suggesting a larger positive valency than other two $\mathrm{TiO}_{2}$. 
As shown above, L-emission of 3d-transiton element is useful not only for probing valence electron state of bonding electrons by L $\alpha, \beta$ but also for charge state (valency) analysis by Ll, $\eta$. SXES-SEM analysis of battery cathode materials which show particular characters depending on different charge states will be also presented.

References:

[1] M Terauchi, et al., Microscopy and Microanalysis 20 (2014), 629.

[2] T Imazono, et al., Applied Optics 51 (2012), 2351.

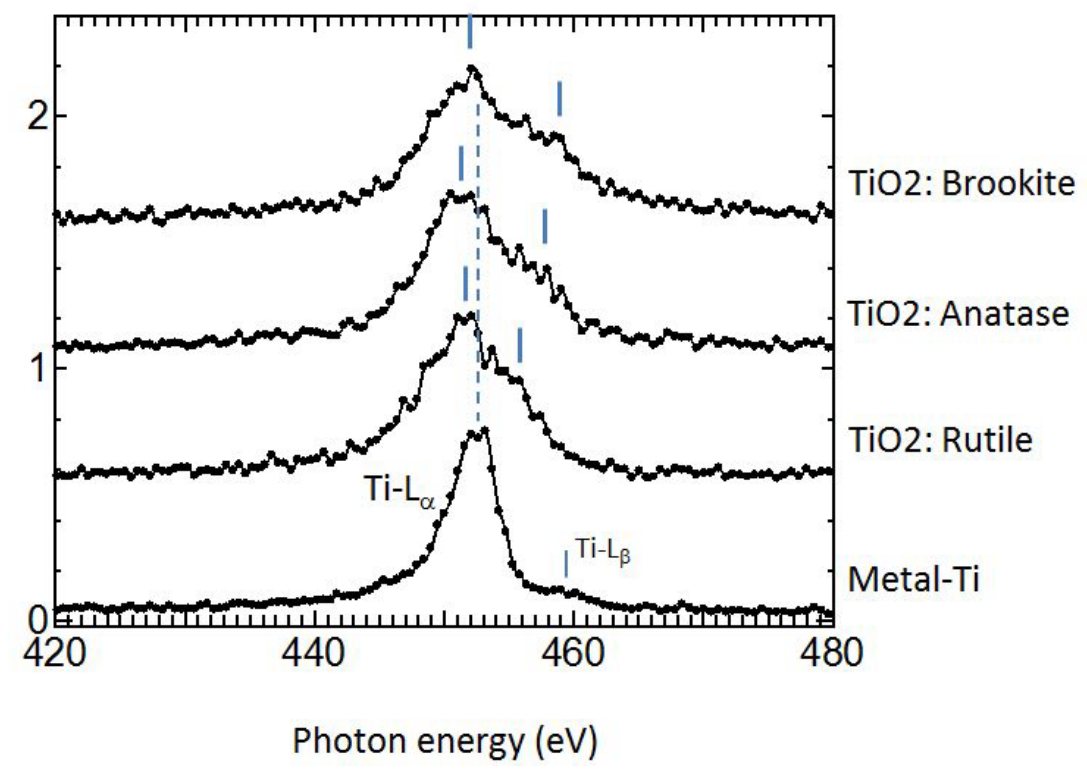

Figure 1. Ti-L $\alpha, \beta$ emission spectra obtained from bulk $\mathrm{TiO}_{2}$ specimens of rutile, and anatase, brookite as well as metal-Ti by using our SXES-SEM with a grating for 300-2200 eV [2].

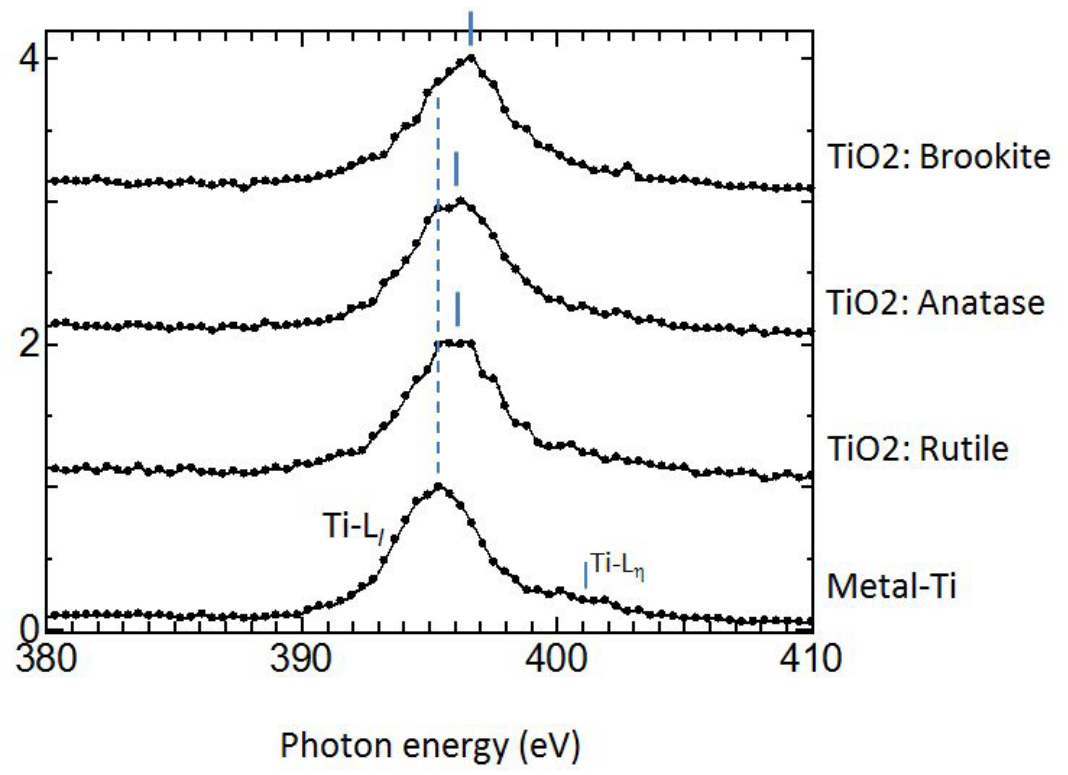

Figure 2. Ti-Ll, $\eta$ emission spectra of bulk $\mathrm{TiO}_{2}$ of rutile, anatase, and brookite, as well as metal-Ti. 